\title{
An Electrical Model of the Cell-Electrode Interface for High-density Microelectrode Arrays
}

\author{
Neil Joye, Alexandre Schmid, Member, IEEE, and Yusuf Leblebici, Senior Member, IEEE
}

\begin{abstract}
A point-contact model is presented, and an areacontact model has been analytically derived in order to model the electrical characteristic of the cell-electrode interface of high-density neuron cultures. The area-contact model is presented as a model more suitable for subcellular multielectrode resolution, which is a requisite for modeling and simulating the electrical behavior of novel high-density microelectrode arrays. Furthermore, when the electrode is aligned and centered with the cell, an optimum electrode diameter for recording the electrical activity of neural cells can be analytically derived, which is between 7-8 $\mu \mathrm{m}$ with a typical load capacitance of $10 \mathrm{pF}$.
\end{abstract}

\section{INTRODUCTION}

$\mathrm{M}$ ICROELECTRODE arrays (MEAs) have become an essential tool both in fundamental and applied research, enabling stepping from the observation of the electrical behavior of single neurons toward the simultaneous analysis of population of neural cells [1]. However, one of the main limitations of MEAs is related to their low spatial resolution. Standard MEAs usually contain approximately 60 electrodes with electrode sizes ranging from 10 to $50 \mu \mathrm{m}$, and inter-electrode spacings up to 100 $\mu \mathrm{m}$. These dimensions are much larger than the $10 \mu \mathrm{m}$ typical size of vertebrate neurons used during electrophysiological experiments. However recently, a new generation of CMOS-based MEAs containing a high-density of sensors has emerged [2], [3]. These new devices have a pitch dimension as low as $7.8 \mu \mathrm{m}$ with an electrode diameter of $4.5 \mu \mathrm{m}$. It is therefore now possible to carry out experiments at subcellular resolution.

The point-contact model [4], which is the standard model used to describe the electrical characteristics of the cellelectrode environment, is not accurate enough to model the electrical properties of the interface at subcellular resolution. This model does not consider the spatial distribution of the different electrical characteristics at the cell-electrode interface. Therefore an area-contact model, where the spatial distribution of the electrical characteristics is taken into account, is needed [4].

Thus, the scope of this work is to develop an area-contact

Manuscript received April 16, 2008. This work has been conducted with the support of the Swiss NSF grant number 205321-116780, and the EPFL STI Seed Grant.

N. Joye, A. Schmid, and Y. Leblebici are with the Microelectronic Systems Laboratroy, Swiss Federal Institute of Technology (EPFL), 1015 Lausanne, Switzerland (phone: +41 -21 6936927; fax: +41 -21 6936959; email: neil.joye@epfl.ch). model of the cell-electrode interface that can be used to describe the electrical properties of high-density MEAs for recording the electrical activity of neural cells. This model is described in Section III, and is compared to a standard point-contact model which is presented in Section II.

\section{POINT-CONTACT Model}

\section{A. Model of the Cell Membrane}

As opposed to the Hodgkin-Huxley model [5], a passive membrane is assumed. This means that no active properties of the membrane are modeled. Thus as depicted in Fig. 1, the equivalent circuit of the attached membrane consists of a resistance $R_{M}$ in parallel with a capacitance $C_{M}$ defined as

$R_{m}=\frac{1}{g_{\text {mem }} A_{c e}}$

$C_{m}=c_{m e m} A_{c e}$

where $A_{c e}$ is the area of the attached membrane, $g_{\text {mem }}=0.3$ $\mathrm{mS} / \mathrm{cm}^{2}$ is the local membrane conductivity [6], and $c_{m e m}=$ $1 \mu \mathrm{F} / \mathrm{cm}^{2}$ is the membrane capacity per unit area. In this paper, a typical cell diameter of $10 \mu \mathrm{m}$ is considered.

\section{B. Model of the Cell-Electrode Interface}

As in [7], the equivalent circuit of the cell-electrode junction is depicted in Fig. 1 . The sealing resistance $R_{\text {seal }}$ represents the resistance between the cleft and the surrounding solution. It has been described in [4] as

$R_{\text {seal }}=\frac{\rho_{s}}{\theta \pi d}$

where $\rho_{s}$ is the resistivity of the electrolyte ( $1 \Omega \mathrm{m}$ for typical solutions), $d$ is the cell-electrode distance, and $\theta$ is a correction factor related to geometry $(\theta=5-8)$.

$C_{h d}$ (cell membrane-electrolyte interface capacitance) models the charge region, also called the electrical double layer, which is formed in the electrolyte at the interface with a neural cell. From the triple-layer model [8], the capacitance $C_{h d}$ is defined as the series of three capacitances: $C_{h 1}, C_{h 2}$ and $C_{d} . C_{h 1}$ is the capacitance between the membrane surface and the first-layer of non-hydrated ions (the Inner Helmholtz Plane); $C_{h 2}$ is the capacitance between the layer of non-hydrated ions and the second layer of hydrated ions (the Outer Helmholtz Plane); and $C_{d}$ is the diffuse layer capacitance (also known as the Gouy-Chapman capacitance). These three capacitances $C_{h 1}, C_{h 2}$ and $C_{d}$ are defined as 


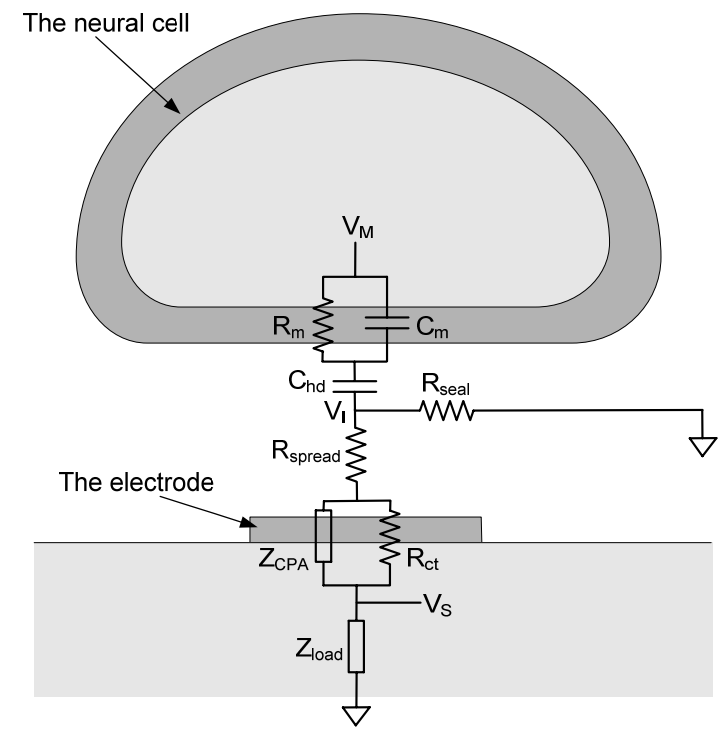

Fig. 1. Point-contact model of the cell-electrode interface (not to scale).

$$
\begin{aligned}
& C_{h 1}=\frac{\varepsilon_{0} \varepsilon_{I H P}}{d_{I H P}} A_{c e} \\
& C_{h 2}=\frac{\varepsilon_{0} \varepsilon_{O H P}}{d_{O H P}-d_{I H P}} A_{c e} \\
& C_{d}=\frac{q \sqrt{2 \varepsilon_{0} \varepsilon_{d} k T z^{2} n^{0} N}}{k T} A_{c e}
\end{aligned}
$$

where $\varepsilon_{0}$ is the dielectric permittivity of free space; $\varepsilon_{I H P}$ and $\varepsilon_{O H P}$ are respectively the Inner and Outer Helmholtz Plane relative dielectric constant; $d_{I H P}$ is the distance of the Inner Helmholtz Plane to the membrane; $d_{O H P}$ is the distance of the Outer Helmholtz Plane to the membrane; $\varepsilon_{d}$ is the diffuse layer relative dielectric constant; $k$ is Boltzmann's constant; $T$ is the absolute temperature; $q$ is the electron charge; $z$ is the valence of ions in solution; $n^{0}$ is the bulk concentration of ions in solution; and $N$ is Avogadro's number.

\section{Model of the Electrode}

The equivalent circuit model of the electrode is described in Fig. 1. It includes a constant phase angle impedance $Z_{C P A}$, which represents the interface capacitance, in parallel with a charge transfer resistance $R_{c t}$, both in series with a spreading resistance $R_{\text {spread }}$. The Warburg impedance due to diffusion of the chemical reactants in solution is not included in this model. It is negligible for the materials and frequency range used in electrophysiological experiments.

The constant phase angle impedance $Z_{C P A}$ is described as

$$
Z_{C P A}=\frac{1}{\left(j \omega C_{d l}\right)^{n}}
$$

where $C_{d l}$ is the double layer capacitance as described in (4)(6), and $n$ is an empirical factor between 0 and 1 representing the surface irregularities.

The charge-transfer resistance $R_{c t}$ represents the faradic process where charges transfer between the electrode and the electrolyte by means of oxidation-reduction reactions. For small values of overpotential during a faradic charge transfer, $R_{c t}$ may be expressed as

$$
R_{c t}=\frac{k T}{q} \frac{1}{z J_{0} A_{e l}}
$$

where $z$ is the number of electrons involved in the oxidationreduction reaction, $J_{0}$ is the equilibrium exchange current density, and $A_{e l}$ is the electrode surface.

The spreading resistance $R_{\text {spread }}$ represents the resistance to the current spreading from the electrode to the counter electrode. Assuming that the counter electrode is infinitely large, the spreading resistance is given in [9] by

$$
R_{\text {spread }}=\frac{\rho_{s}}{4 R_{e l}}
$$

for circular electrodes ( $R_{e l}$ is the radius of the electrode).

\section{Transfer Function of the System}

The transfer function $H(j \omega)=V_{S}(j \omega) / V_{M}(j \omega)$ of the system described in Fig. 1 is established as

$$
\begin{aligned}
& H(j \omega)=\frac{R_{\text {seal }} Z_{\text {load }}}{Z_{m} R_{\text {seal }}+\left(Z_{m}+R_{\text {seal }}\right)\left(Z_{\text {load }}+Z_{\text {el }}\right)} \\
& Z_{m}(j \omega)=\frac{j \omega R_{m} C_{h d}}{\left(1+j \omega R_{m} C_{m}\right)\left(j \omega C_{h d}\right)} \\
& Z_{e l}(j \omega)=\frac{\left(R_{c t}+R_{\text {spread }}\right)+R_{c t} R_{\text {spread }}\left(j \omega C_{d l}\right)^{n}}{1+R_{c t}\left(j \omega C_{d l}\right)^{n}}
\end{aligned}
$$

where $Z_{m}$ is the membrane impedance with $C_{h d}, Z_{e l}$ is the electrode impedance, and $Z_{\text {load }}$ is the load impedance. The load impedance represents the input impedance of the first amplification stage. It is typically a $1-20 \mathrm{pF}$ capacitance.

The Bode plots for the amplitude and phase of $H(j \omega)$ are simulated under Matlab using the numerical values detailed in the following. For $C_{h d}$, the values given in [7] are considered. In particular, it is assumed that $\varepsilon_{I H P}=6, \varepsilon_{O H P}=$ $32, d_{I H P}=0.3 \mathrm{~nm}, d_{O H P}=0.7 \mathrm{~nm}, z=1, \mathrm{~T}=300 \mathrm{~K}$, and $n^{0}=$ $150 \mathrm{mM}$. For a Pt electrode, the values given in [9] are considered for $Z_{C P A}$ and $R_{C t}$; $d_{O H P}$ is equal to $0.5 \mathrm{~nm}, d_{I H P}$ is neglected, $\varepsilon_{O H P}=78, \mathrm{z}=4, n^{0}=150 \mathrm{mM}$, and $\mathrm{n}=0.9$. Finally, a typical load capacitance of $10 \mathrm{pF}$ is taken into account.

The Bode plots for the amplitude and phase of $H(j \omega)$ are simulated with a typical cell-electrode distance of $70 \mathrm{~nm}$ [7] and a $5 \mu \mathrm{m}$ diameter circular electrode, as depicted in Fig. 2. The value of the scaling factor $\theta$ used to determine the sealing resistance in (3) is fixed to 5.78 as described in [10]. The voltage drop across the electrode is almost negligible for a frequency range of $10 \mathrm{~Hz}$ to $10 \mathrm{kHz}$, which corresponds approximately to the frequency range of neural activity. Moreover, the voltage attenuation between the potential that is sensed $V_{S}(j \omega)$ and the intracellular potential $V_{M}(j \omega)$ has a large variation in amplitude depending on the frequency of the neural signal. In the configuration chosen for Fig. 2, $V_{S}(j \omega)$ is about 4 orders of magnitude smaller than 

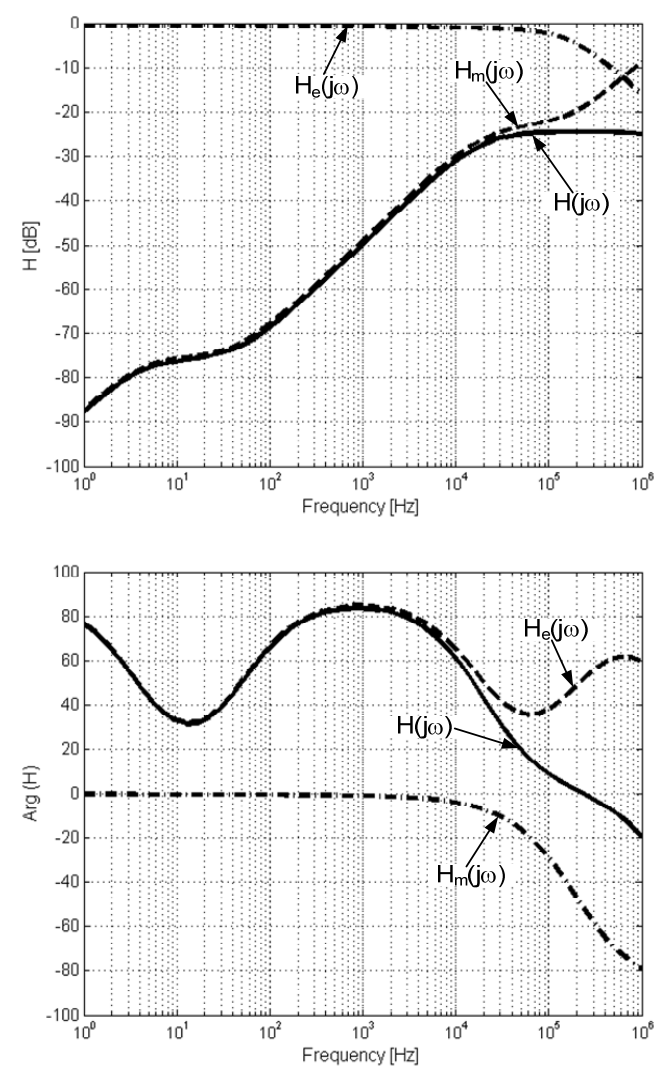

Fig. 2. Bode plots for the amplitude and phase of $H(j \omega)$. A cell-electrode distance of $70 \mathrm{~nm}$ and an electrode diameter of $5 \mu \mathrm{m}$ are considered. $H(j \omega)$ $=H_{m}(j \omega) H_{e}(j \omega)=\mathrm{V}_{\mathrm{S}}(\mathrm{j} \omega) / \mathrm{V}_{\mathrm{M}}(\mathrm{j} \omega)$ is the transfer function of the system, with $H_{m}(j \omega)=V_{I}(j \omega) / V_{M}(j \omega)$ and $H_{e}(j \omega)=V_{S}(j \omega) / V_{I}(j \omega)$.

$V_{M}(j \omega)$ for a frequency of $10 \mathrm{~Hz}$, and only 2 orders of magnitude smaller for a frequency of $3 \mathrm{kHz}$. Therefore, in the frequency range of interest, the cell-electrode system behaves as a high-pass filter.

Furthermore, the amplitude of the transfer function $H(j \omega)$ versus the cell-electrode distance is depicted in Fig. 3 for various frequencies. As expected, these results emphasize the importance of having a small cell-electrode distance in order to obtain a large electrical coupling between $V_{S}(j \omega)$ and $V_{M}(j \omega)$. Typically, the cell-electrode distance can be as small as $10 \mathrm{~nm}$ for a strong adhesion of the cell to the electrode surface, and can reach values as large as 100 to $200 \mathrm{~nm}$ for weak adhesion conditions [6], [7].

\section{AREA-Contact Model}

\section{A. Cell-Electrode Interface Voltage}

First, the voltage $V_{I}(r, j \omega)$ at the cell-electrode interface, where $r$ is the distance to the center of the cell, is established. As a first approximation step, the electrode is not taken into account, as depicted in Fig. 4. Moreover, the assumption that the electrical potential is constant across the cytoplasm is made. Therefore in the Laplace domain, the attached membrane can be described as

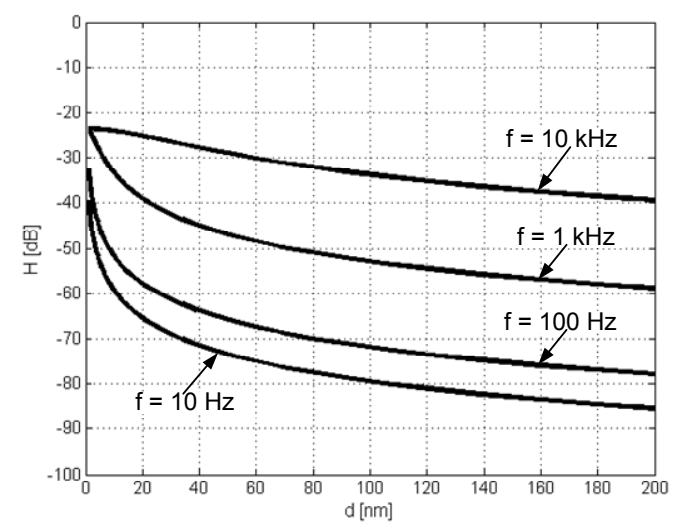

Fig. 3. Amplitude of the transfer function $H(j \omega)$ versus the cell-electrode distance for different frequencies of $V_{M}$.

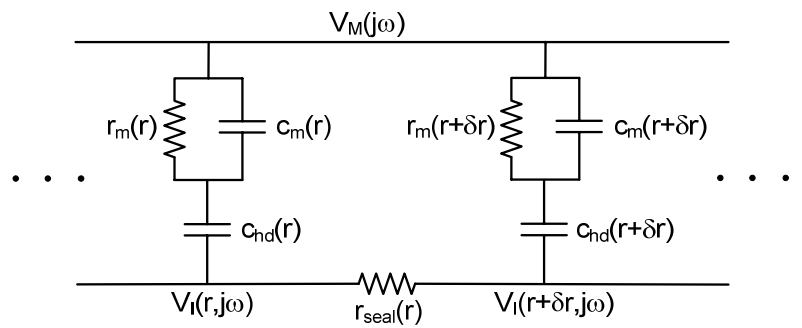

Fig. 4. Area-contact model of the attached cell membrane, where $V_{M}(j \omega)$ is the intracellular potential, and $V_{I}(r, j \omega)$ is the potential at the cellelectrode interface at a distance $r$ from the center of the cell.

$$
\begin{aligned}
\frac{V_{I}(r, s)-V_{I}(r-\partial r, s)}{r_{\text {seal }}(r)}+\frac{V_{M}(s)-V_{I}(r, s)}{z_{m}(r)}= \\
\frac{V_{I}(r+\partial r, s)-V_{I}(r, s)}{r_{\text {seal }}(r)}
\end{aligned}
$$

where $r_{\text {seal }}(r)$ and $z_{m}(r)$ are respectively the infinitesimal radius-dependent values of $R_{\text {seal }}$ and $Z_{m}$.

With the approximation given in (14), the cell-electrode interface can be expressed as the first order liner equation given in (15).

$$
\begin{aligned}
\partial V_{I}(r, s)=V_{I}(r, s)-V_{I}(r-\partial r, s) & \\
& =V_{I}(r+\partial r, s)-V_{I}(r, s) \\
\frac{\partial V_{I}(r, s)}{\partial r}+\operatorname{arV}_{I}(r, s)= & \operatorname{arV}_{M}(s)
\end{aligned}
$$

where $a$ is

$a=\frac{\rho_{s}\left(g_{\text {mem }}+s c_{\text {mem }}\right)}{2 d}$

With the initial condition $V\left(R_{c e}, s\right)=0$, where $R_{c e}$ is the radius of the cell, the solution of this equation is

$V_{I}(r, s)=V_{M}(s)\left(1-e^{a\left(r^{2}-R_{c e}^{2}\right)}\right)$

The distribution of the cell-electrode interface voltage $V_{I}(r, s)$ for various frequencies is shown in Fig. 5. The electrical property values that where used for the pointcontact simulation depicted in Fig. 2 is considered as well again. As expected, $V_{I}(r, s)$ is maximum at the center of the cell and is minimum at the edge of the cell. 


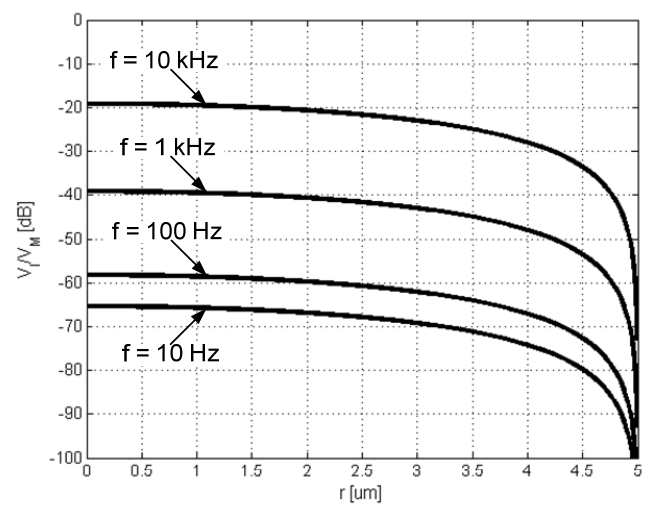

Fig. 5. $V_{I} / V_{M}$ versus the distance from the center of the cell for different frequencies of $V_{M}$ corresponding to the frequency range of neural activity.

\section{B. Transfer Function of the System for an Electrode Centered with the Cell}

In the area-contact model, the electrode can be described as presented in Fig. 6. Thus, $V_{S}(s)$ can be expressed as

$$
\begin{aligned}
& V_{s}(s)= Z_{\text {load }} \int_{0}^{R_{e l}} \frac{V_{I}(r, s)-V_{s}(s)}{z_{e l}(r, s)} \\
& V_{s}(s)=\frac{\pi\left(1+r_{t}\left(s c_{d}\right)^{n}\right)}{s C_{\text {load }} r_{t}+R_{e l}^{2} \pi\left(1+r_{t}\left(s c_{d}\right)^{n}\right)} \\
& \\
& {\left[R_{e l}^{2}+\frac{e^{-a R_{c e}^{2}}-e^{a\left(\operatorname{R}_{e l}^{2}-R_{c e}^{2}\right)}}{a}\right] V_{M}(s) }
\end{aligned}
$$

where $z_{e l}(r, s)$ is the infinitesimal value of $Z_{e l}$.

$V_{S}(s) / V_{M}(s)$ versus the electrode radius has been plotted in Fig. 7 for three different cell-electrode distances, considering the same physical and electrical values that were used in Fig. 2 and Fig. 5. For electrodes diameters smaller than $1 \mu \mathrm{m}$, the transfer function $H(s)$ can be more than 20 $\mathrm{dB}$ smaller than for diameters around $8-10 \mu \mathrm{m}$. Thus, the size of the electrode must be increased. However if the electrode size is too large, $V_{S}(s)$ slightly decreases due to the decrease of $V_{I}(r, s)$ at the edge of the cell as depicted in Fig. 5. An optimum electrode radius can be clearly identified between $3.5 \mu \mathrm{m}$ and $4 \mu \mathrm{m}$.

\section{CONCLUSION}

An adapted point-contact model is presented, and an areacontact model is analytically derived, enabling to model the electrical behavior of the cell-electrode interface of highdensity neuron cultures during electrical activity recording. The area-contact model is presented as a model more suitable for subcellular resolution, which is a requisite for modeling and simulating the electrical behavior of novel high-density MEAs.

Finally, electrical measurements will have to be performed on fabricated MEAs in order to confirm the simulation results obtained with the models presented in this paper.

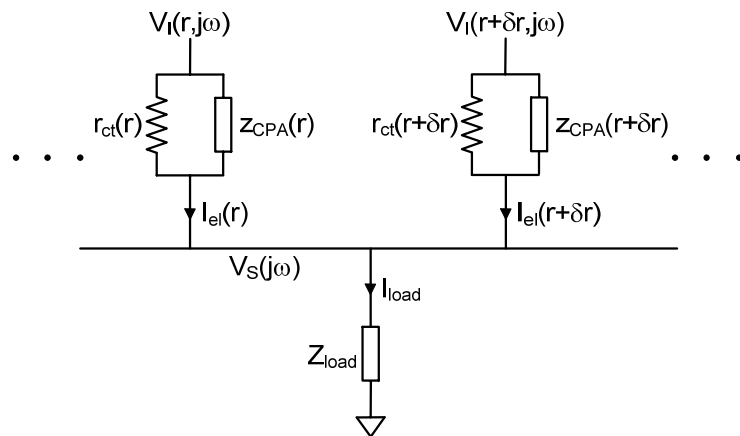

Fig. 6. Area-contact model of the electrode with the load impedance ( $\mathrm{R}_{\text {spread }}$ is neglected).

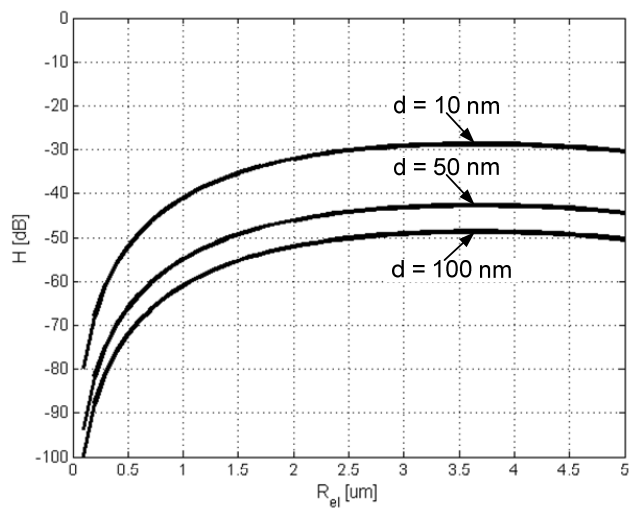

Fig. 7. Amplitude of the transfer function $H(s)=V_{S}(s) / V_{M}(s)$ versus the electrode radius for different cell-electrode distances. A frequency of 1 $\mathrm{kHz}$ is used.

\section{REFERENCES}

[1] S. Marom and G. Shahaf, "Development, learning and memory in large random networks of cortical neurons: lessons beyond anatomy," Quarterly Reviews of Biophysics, vol. 35, no. 1, pp. 63-87, May 2002.

[2] U. Frey et al., "An 11k-electrode 126-channel high-density microelectrode array to interact with electrogenic cells," in Proc. IEEE ISSCC 2007, pp. 158-593.

[3] B. Eversmann et al., "A $128 \times 128$ CMOS biosensor array for extracellular recording of neural activity," IEEE J. Solid-State Circuits, vol. 38, no. 12, pp. 2306-2317, Dec. 2003.

[4] P. Fromherz, "Neuroelectronic interfacing: semiconductor chips with ion channels, nerve cells, and brain," in Nanoelectronics and Information Technology, Berlin: Rainer Waser, Wiley-VCH, 2003, pp. 781-810.

[5] A. L. Hodgkin and A. F. Huxley, "A quantitative description of membrane current and its application to conduction and excitation in nerve," J. Physiol., vol. 117, pp. 500-544, Aug. 1952.

[6] J. R. Buitenweg, W. L. C. Rutten, and E. Marani, "Geometry-based finite-element modeling of the electrical contact between a cultured neuron and a microelectrode," IEEE Trans. Biomed. Eng., vol. 50, no. 4, pp. 501-509, Apr. 2003.

[7] P. Massobrio, G. Massobrio, and S. Martinoia, "Multi-program approach for simulating recorded extracellular signals generated by neurons coupled to microelectrode arrays," Neurocomputing, vol. 70, pp. 2467-2476, Aug. 2007.

[8] J. O. M. Bockris and A. K. N. Reddy, Modern Electrochemistry. New York: Plenum Press, 1970, vol. 2.

[9] W. Franks, I. Schenker, P. Schmutz, and A. Hierlemann, "Impedance characterization and modeling of electrodes for biomedical applications," IEEE Trans. Biomed. Eng., vol. 52, no. 7, pp. 12951302 , July 2003.

[10] D. Braun and P. Fromherz, "Imaging neuronal seal resistance on silicon chip using fluorescent voltage-sensitive dye," Biophysical Journal, vol. 87, pp. 1351-1359, Aug. 2004. 